\title{
Gairdner presents cutting-edge cancer research
}

$\mathrm{H}$ ow do you treat a patient with cancer once the tumour has metastasized to multiple organs? Is it possible to prevent relapse once a patient with leukemia is in remission? Leading cancer researchers from Canada and the United States pitched experimental findings and debated untested theories on these and other questions during the recent Gairdner Symposium on new advances in the biology and treatment of cancer at the University of Toronto.

The celebrated winner of one of this year's Canada Gairdner International Awards, James Allison, professor and chair of the Department of Immunology at the University of Texas MD Anderson Cancer Center in Houston, is intimately acquainted with these sorts of debates. Allison spent nearly two decades fighting skeptics over his innovative ideas for treating cancer by harnessing the immune system to attack tumours. When other presenters at a roundtable discussion said that cancer immunotherapy is now poised to enter mainstream clinical oncology, he agreed, saying, "We don't have to hide anymore."

The Canada Gairdner awards, the country's leading science prizes, include $\$ 100000$ to each of five scientists who have moved their fields forward. Allison won for discovering that receptors on the surface of $\mathrm{T}$ cells keep the $\mathrm{T}$ cells in check, preventing an immune response to cancer. Beginning in the mid-1990s, he focused on one particular receptor, cytotoxic T-lymphocyte-associated antigen 4 (CTLA4 ), and figured out that blocking it with an antibody — anti-CTLA-4 — could turn on the body's immune defences.

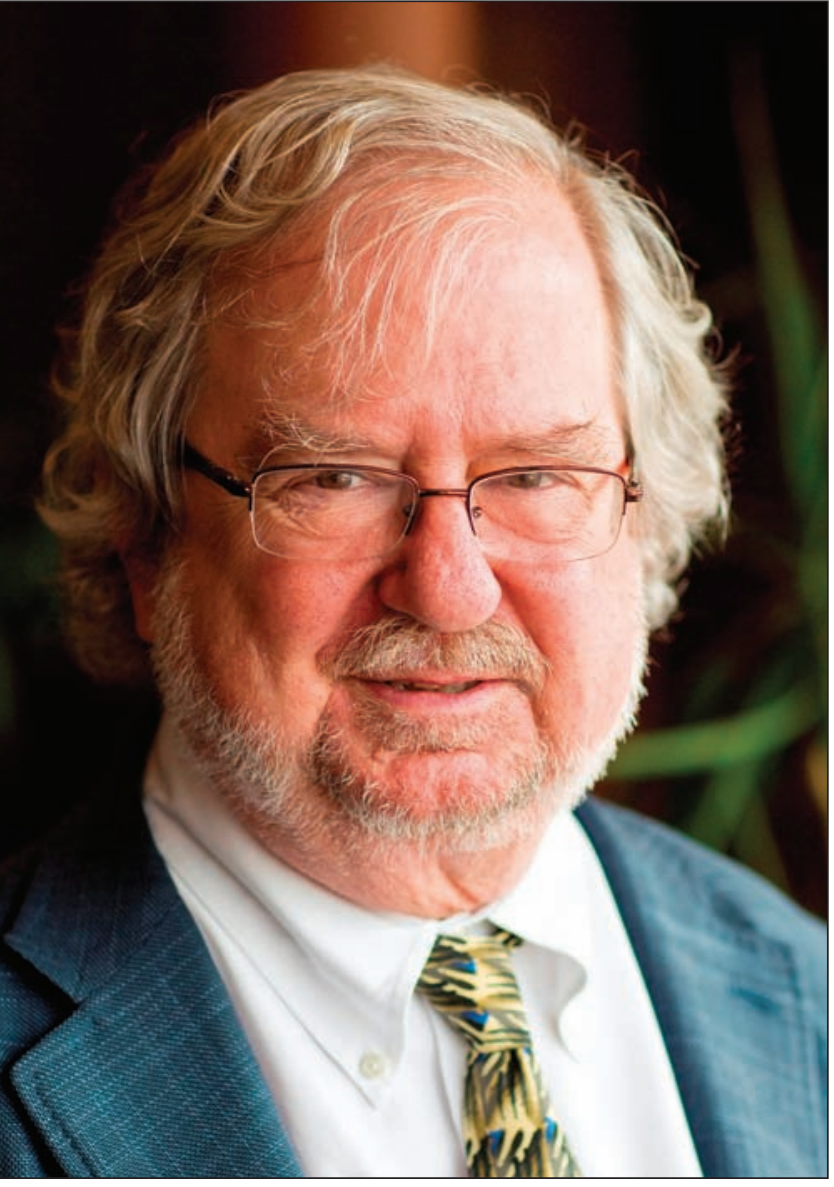

James Allison won a $\$ 100000$ Canada Gairdner award for discovering that receptors on the surface of $T$ cells keep the $T$ cells in heck, preventing an immune response to cancer.

The strategy he pioneered, known as immune checkpoint blockade, has led to therapeutic breakthroughs for patients with late-stage and metastatic cancers for whom few treatments are available. Anti-CTLA-4, now known as ipilimumab, was approved in Canada this year as a first-line therapy for patients with unresectable or metastatic melanoma.

The Gairdner Foundation is not alone in taking notice. Science Magazine named cancer immunotherapy its "Breakthrough of the Year" in 2013, and on Nov. 9, Allison will take home US\$3 million as a winner of the Breakthrough Prize in Life Sciences.

Anti-CTLA-4 "opened an entire new way of thinking about cancer therapy," Dr. Suzanne Topalian, professor of surgery and oncology at Johns Hopkins University in Baltimore, told the Oct. 31 symposium. Industry is also involved Bristol-Myers Squibb bought the small company that developed anti-CTLA-4 for $\$ 2.4$ billion in 2009 ; Goldman Sachs estimated last year that cancer immunotherapy will have a market potential of US\$10-15 billion by 2025 .

One of the most striking aspects of using the immune system is that once activated, it stays on. Topalian described this phenomenon in a 57-year-old man with advanced kidney cancer and liver metastases who entered a trial she and her colleagues are running, after surgery and four attempts at drug regimens failed to stop his tumour from growing. The trial uses the experimental drug, nivolumab, which blocks another receptor that inhibits T cells. The man's tumour regressed while he was taking the drug, and though the drug was stopped after two years, the cancer did not regrow.

"Now this is very unusual in oncology, to stop a drug and to be watching a tumour every two months when you're scanning patients, and it's just sitting there and not changing," Topalian said. "But we have seen this over and over again in our patients."

However, toxicity is a major problem. Most patients experience adverse effects with ipilimumab, some quite serious, and in a published study of patients with previously treated metastatic melanoma, 14 deaths were related to the drug for about a $2 \%$ drugrelated mortality rate (NEJM 2010; 363:711-23). 
Another potential therapeutic agent discussed at the symposium was the use of genetically engineered viruses called oncolytic viruses that might work without causing serious adverse effects. At least, that's what is seen in mice. "The oncolytic paradigm is: a virus goes in, finds the tumours, grows within them, destroys them and then is cleared from the body," John Bell, senior scientist, Cancer Therapeutics Program, Ottawa Hospital Research Institute told the symposium. If all goes as planned, the virus replicates selectively in the tumour and does not damage normal tissues.

As head of the Canadian Oncolytic Virus Consortium, Bell leads a group that took a virus found on sandflies in Brazil, did "a lot of engineering to make it more potent," and tested it in mice and monkeys. Those tests led to approval for a first-in-human clinical trial: patients with metastatic colorectal cancer and metastatic non-small-cell lung cancer can enroll in four cities across Canada as of this month.

Though his research remains highly controversial, Bell sees the future as bright. He reached out to the students, urging them to think about oncolytic viruses, as "very cool machines that we can make do the tricks we want," and encouraging them to enter the field. Miriam Shuchman, Toronto

CMAJ 2014. DOI:10.1503/cmaj.109-4939 